\title{
Formulation of the Problem of Maximum Clique Determination in Non-Oriented Graphs
}

\author{
S. V. Listrovoy ${ }^{1}$, O. V. Golovko ${ }^{1}$, V. M. Butenko ${ }^{1 *}$, M. V. Ushakov ${ }^{1}$ \\ ${ }^{I}$ Ukrainian State University of Railway Transport \\ *Corresponding author E-mail: butenko@kart.edu.ua
}

\begin{abstract}
In the train traffic organization, large amount of information should be processed in real time. Thereby in complicated dispatching systems, rational use of resources is needed. To perform this work a model of a management system is constructed and it is represented as a sparse graph. Further optimization of the model requires solving the Maximum Clique Problem (MCP) for the less time than the exponential time. This article contains two procedures for solving the task for subexponential time. Procedure B accurately estimates the size of the maximum clique in the graph and performs the sorting of the vertices, in such a way that the vertices that are not exactly in the maximum clique can be rejected. Procedure A, in fact, finds cliques of the maximum size in a given graph, using the procedure B. The main advantage of this method is that using it is expedient in real time for sufficiently large graphs, which in turn is important for the construction of control systems.
\end{abstract}

Keywords: cliques in non-oriented graph; cost optimization tools; maximum clique problem; railway infrastructure; safety of control systems

\section{Introduction}

The use of ceramic cutting tools from nano-structured refractory compounds [1] partially improves the performance of railway transport. However, in the operation of industry management systems, the problems of forecasting the technical condition of railway automation equipment in the conditions of limited statistical data on the safety of information-control systems are acute [2]. Functional safety is defined in the paper [3] and imposes certain restrictions on the operation of new railway automation systems [4]. Control, signalling and communication systems received new requirements in the paper [5]. One of the ways to improve the safety and accuracy of determining technical parameters for determining the parameters of motion and, as a consequence, improving the prediction of the technical state - the use of video analysis methods [6]. The use of video analysis methods with increasing accuracy leads to a significant increase in information flows and the construction of distributed computer networks. This prompted specialists to search for opportunities to optimize resources, both in the technical and in the information management subsystem. Simulation of the work of a local scheduler on the basis of solving problems of nonlinear Boolean programming has already been considered by the authors in [7]. This will optimize computing resources and prevent premature investments in hardware infrastructure.

The model of control systems under consideration is equivalent to formal definitions of sparse graphs. It can be replaced by the Maximum Clique Problem (MCP), which is one of the known hard-tosolve problems in graph theory. Solutions exist, but they take time exponentially depending on the size of the graph. And so far no algorithms have been found that solve it in polynomial time with the possibility of parallel computations. Thus, the current direction of the MCP research is the development of new approaches for finding exact and approximate solutions taking into account the peculiarities of graphs arising in applications.

The objective of the work was to develop a method for determining maximal cliques in non-oriented graphs with a small time complexity.

To achieve this objective, there was a need for the following:

- enter procedure $\mathrm{B}$, which allows determining the estimates on top of the sizes of cliques in the graphs;

- enter procedure A, which allows using procedure B to generate cliques on the basis of each vertex of the graph and then select the largest clique in the graph.

\section{Determination the Problem}

We construct the solution of the MCP problem on the basis of obtaining the upper estimates of the sizes of the cliques in the graph. The basis is the rather obvious assertion 1 .

Assertion 1. Suppose that a clique of size $\mathrm{k}$ is presented in the graph $\mathrm{G}(\mathrm{V}, \mathrm{E})$. Then the number of vertices $\mathrm{i}$ with degree $d_{i} \geq k-1$ must be at least $k$. Thus, we have an upper bound on the size of the maximal clique $\Delta_{\max }=k$ in the graph $\mathrm{G}(\mathrm{V}, \mathrm{E})$.

Let $\mathrm{G}(\mathrm{V}, \mathrm{E})$ be the graph containing $\mathrm{n}$ vertices. We note at once that the graph is not complete. This is easily verified and the solution of the maximum clique problem, in this case, is trivial. Hence the size of the maximum clique is strictly less than $\mathrm{n}$. But this is a rough estimate. For clarity, we construct $\mathrm{n}$ subgraphs corresponding to the vertices of the graph. Namely: choose the vertex $i=1$, select all the vertices associated with it. Next, we connect all vertices by edges in accordance with the constraints in the original graph $\mathrm{G}(\mathrm{V}, \mathrm{E})$. We obtain a subgraph $\mathrm{G} 1$ without a vertex $\mathrm{I}=1$, we similarly construct subgraphs $\mathrm{G}_{2}, \mathrm{G}_{3}, \ldots, \mathrm{G}_{\mathrm{n}}$.

Summarizing, the formation of an arbitrary subgraph $\mathrm{Gi}$ on the basis of an arbitrary vertex $i$ consists in isolating a subset of vertices connected with the vertex i. Next, we connect them by edges 
in accordance with the vertex connections that took place in the graph G. Obviously, the maximum size clique is presented in the original graph $\mathrm{G}(\mathrm{V}, \mathrm{E})$ must be present in one of the subgraphs $\mathrm{Gi}$. We construct procedure B to determine the upper estimates for the sizes of the cliques in the subgraphs $G_{i}$. Suppose that the graph $G_{i}$ is given with its vertices sorted in decreasing order of powers $d_{v}$. That is, vertices having the same powers $d_{r}$ are joined into subsets of $\left\{\alpha^{r}\right\}$ with cardinality $\left|\alpha^{r}\right|=p_{r}$ (where $\alpha^{r}$ are the numbers of the corresponding vertices in $G_{i}$ ), and $p_{r}$ the number of vertices in $G_{i}$ with the same powers of $d_{r}$. We denote the total number of such subsets $\gamma$. We sort the sequence of subsets of vertices $\left\{\alpha^{r}\right\}$ in such a way that they satisfy the inequalities:

$d_{r=1}>d_{r=2}>\cdots>d_{r=\gamma}$,

where $d_{r}$ is the degree of the vertices of the subset $\left\{\alpha^{r}\right\}$. Then we form an ordered sequence:

$\left\{\left\{\alpha^{r=1}\right\},\left\{\alpha^{r=2}\right\}, \ldots\left\{\alpha^{r=\gamma}\right\}\right\}$

Ordered sequence (2) will be stored as a list $S_{\gamma}$. We propose the following procedure for determining the upper bound $\Delta_{\max }$ on the list $S_{j}$ on the basis of Assertion 1

Procedure B definitions $\Delta_{\max }$

Step 1. Sorting vertices and forming subsets $\left\{\alpha^{r}\right\}$.

Step 2. $r:=1$.

Step 3. Check the inequality:

$\sum_{i=1}^{r-1} P_{i}+P_{r} \geq d_{r}+1$

if the inequality is satisfied, then $\Delta_{\max }:=d_{r}+1$ and the procedure ends, otherwise, we perform the next step.

Step 4. $r:=r+1$ and proceed to step 3 .

Note that in an inequality whose truth is verified in step 3 for $\mathrm{r}=1$, the sum is:

$\sum_{i=1}^{r-1} P_{i}=0$.

An example of a definition $\Delta_{\max }$ for the graph shown in Fig. 1. When sorting vertices (Table 1), four subsets are formed: $\alpha^{r=1}=$ \{1\} with powers $d_{r}=5, \alpha^{r=2}=\{3,5,6\}$ with powers $d_{r}=4$, $\alpha^{r=3}=\{2,7,8\}$ with powers $d_{r}=3$, and $\alpha^{r=4}=\{4\}$, with powers $d_{r}=2$. Accordingly, the powers of the resulting subsets $P_{r=1}=1$, $P_{r=2}=3, P_{r=3}=3, P_{r=4}=1$. Assign $\mathrm{r}:=1$ to investigate the subset $\alpha^{r=3}$ and check the inequality (3), in this case it has the form $0+1 \geq 5+1$.

Inequality is not satisfied, therefore, further $r:=r+1=1+1=$ 2 and go to the set $\alpha^{r=2}$. We check the inequality $P_{r=1}+P_{r=2} \geq$ $d_{r=2}+1$ and get $1+3 \geq 4+1$. This inequality is also not fulfilled. Then follows step $3 r:=r+1=2+1=3$ and in step 3 go to the set $\alpha^{r=3}$. We check the inequality $P_{r=1}+P_{r=2}+P_{r=3} \geq d_{r=3}+1$, we get $1+3+3 \geq 3+1$. Since this inequality is satisfied, hence $\Delta_{\max }=4$. Thus, in the graph, the maximum clique cannot exceed the size equal to 4. If there was a larger clique in the graph, for example, size 5 , then the number of vertices with a power of 4 should be greater than or equal to five, and for the size of the clique 4 , the number of vertices with degrees 3 must be greater than or equal to four. The results of sorting the vertices of the graph are given in Table 1 .

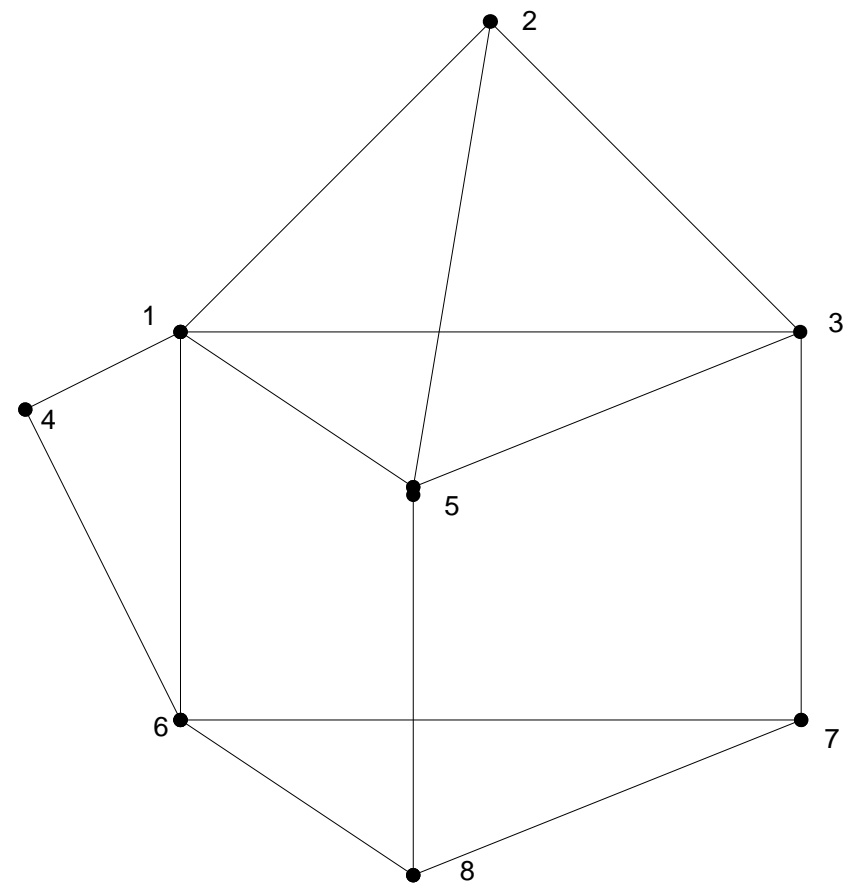

Fig. 1: The graph G.

\begin{tabular}{|c|c|c|c|}
\multicolumn{2}{|c}{ Table 1: Results of sorting the vertices of a graph } \\
\hline \multirow{2}{*}{ Sets $\left\{\alpha^{r}\right\}$} & Vertices $i$ & $\begin{array}{c}\text { Number of } \\
\text { edges } d_{r}\end{array}$ & $\begin{array}{c}\text { Cardinality } P_{r} \text { of } \\
\text { the } \operatorname{set}\left\{\alpha^{\prime}\right\}\end{array}$ \\
\hline Set $\alpha^{r=1}$ & 1 & 5 & \multirow{2}{*}{$P_{r=1}=1$} \\
\hline Set $\alpha^{r=1}$ & 3 & 4 & \multirow{2}{*}{$P_{r=2}=3$} \\
\cline { 2 - 3 } & 5 & 4 & \multirow{2}{*}{$P_{r=3}=3$} \\
\hline Set $\alpha^{r=1}$ & 6 & 4 & \\
\cline { 2 - 3 } & 2 & 3 & $P_{r=4}=1$ \\
\hline
\end{tabular}

Let the graph $\mathrm{G}$ consist of vertices $\{\mathrm{i}\}, i=(\overline{1, n})$. We define the upper bounds $H=\left\{\Delta_{\text {maxi }}\right\}$ of the sizes of the maximal clique sizes that can be formed on the basis of vertices $\{i\}, i=(\overline{1, n})$. We introduce the notation, let there be $\mathrm{k}$ vertices in the set $H=\left\{\Delta_{\text {maxi }}\right\}$ with estimates $\left\{\Delta_{\text {maxi }}^{*}=k\right\}$, then we denote the subset of these vertices as $h_{\text {max }}=\left\{\Delta_{\text {maxi }}^{*}=k\right\}$. Let's say that a subset $h_{\text {max }}=\left\{\Delta_{\text {maxi }}^{*}=k\right\}$ has a property $v$, if an inequality holds $\Delta_{\text {maxi }}^{*}>\Delta_{\operatorname{maxi}}$, where $\Delta_{\max i}$ is the estimate of the vertices $\epsilon h_{\max }$, and $\Delta_{\text {maxi }}$ is the estimate of the vertices belongs $H$. Then the following assertion holds.

Assertion 2. If in the set $H=\left\{\Delta_{\text {maxi }}\right\}$ obtained for some graph G there is a subset $h_{\max }=\left\{\Delta_{\text {maxi }}^{*}=k\right\}$ satisfying the property $v$, then the size of the maximal clique in the graph is equal to $\left|h_{\text {max }}\right|=\left\{\Delta_{\text {maxi }}^{*}=k\right\}$ and the subset of vertices $\{\mathrm{i}\} \in h_{\text {max }}$, that is, the corresponding $\left\{\Delta_{\text {maxi }}^{*}=k\right\}$ form a given clique.

Assertions 2 are true, since on the basis of all vertices not belonging to $h_{\max }=\left\{\Delta_{\text {maxi }}^{*}=k\right\}$ it is impossible to build cliques large in size than cliques that can be constructed on the basis of vertices belonging to $h_{\text {max }}=\left\{\Delta_{\text {maxi }}^{*}=k\right\}$ (which follows from the property $v)$. The estimates in $h_{\max }=\left\{\Delta_{\text {maxi }}^{*}=k\right\}$ are the same by definition, $\left(\left|h_{\max }\right|=\Delta_{\text {maxi }}^{*}\right)$ therefore it is impossible to construct a larger clique than the set of vertices $\{i\}$, corresponding to $\left\{\Delta_{\text {maxi }}\right\}$ in the graph $\mathrm{G}$.

If in the set $H=\left\{\Delta_{\text {maxi }}\right\}$ obtained for the graph $\mathrm{G}$, there is no subset $h_{\text {max }}=\left\{\Delta_{\text {max }}^{*}\right\}$ satisfying the property $v$, then we sort the vertices of this graph in the decreasing order of the estimates $\Delta_{\text {maxi }}$. We combine vertices with equal estimates $\Delta_{\text {maxi }}$ into subsets $\left\{\lambda^{r}\right\}$ with cardinality $\left|\lambda^{r}\right|=p_{r}$, the total number of such subsets denote as $\beta$. 
We sort the sequence of subsets of vertices $\left\{\lambda^{r}\right\}$ into a sequence in such a way that the estimates corresponding to $\Delta_{\operatorname{maxi}}$ satisfy inequalities:

$\Delta_{\operatorname{maxi}=1}>\Delta_{\max i=2}>\cdots>\Delta_{\max i=\beta}$.

That is, we will compose an ordered sequence:

$\left\{\left\{\lambda^{r=1}\right\},\left\{\lambda^{r=2}\right\}, \ldots\left\{\lambda^{r=\beta}\right\}\right\}$.

Sequence (6) will be stored as a list $S_{\beta}$. Using procedure B, we define a subset of vertices $r^{*}$ for which inequality:

$\sum_{i=1}^{r-1} P_{i}+P_{r} \geq \Delta_{\max r}+1$

After determining the subset of vertices $r^{*}$, remove from the list $S_{\beta}$ all the vertices of the graph $G$ that lie after $r^{*}$ with the edges incident to them.

Assertion 3. If the subgraph $G^{\prime}$ is constructed on the basis of the list $S_{\beta}$ obtained after deleting the vertices for which $r>r^{*}$, then it contains a clique of the maximum size.

The validity of Assertion 3 is fairly obvious, because on the basis of the vertex in the list $S_{\beta}$ after $r^{*}$, it is impossible to build cliques of a size larger than cliques based on the vertices in the list up to $r^{*}$.

\section{Method of Solving the Problem}

Using procedure $B$, we construct the procedure $A$ of forming the maximal clique in an arbitrary graph $G$. The initial data for the operation of procedure $A$ is the graph $G$ itself and the list of vertices of this graph $S$.

Procedure $A$ :

Step 1 . We check whether there are vertices $Y=\{i\}$ in the graph $G$ associated with all vertices of $G$. The set of vertices of $Y$ and the edges incident to them is deleted from the graph and put into the set $U$. After this, we check whether all vertices are removed from the graph $G$. If so, then the procedure ends, since the original graph represents the maximum size clique, otherwise we perform the next step.

Step 2. We check all the vertices that have the same degree in the graph. If so, we check if there is a vertex $\mathrm{j}$ connected to all vertices in $\mathrm{U}$ among the remaining vertices. If so, the procedure ends, since the set of vertices $\{j \cup U\}$ form the maximum clique in the graph, otherwise we perform the next step.

Step 3. Select the vertex i from the list $\mathrm{S}$ and form the subgraph $\mathrm{Gi}$ (Vi, Ei) on the basis of vertices adjacent to i.

We verify that in the subgraph $G_{i}\left(V_{i}, E_{i}\right)$ there are vertices $\mathrm{D}=\{\mathrm{i}\}$ connected with all vertices of the subgraph $G_{i}\left(V_{i}, E_{i}\right)$, and remove the set of vertices $\mathrm{D}$ and the edges incident to them from the graph and place them in the set $U_{i}$, after this verifies whether all vertices are removed from the subgraph $G$. If so, then $\Delta_{\text {maxi }}=\left|V_{I}\right|$ otherwise we perform the next step.

Step 4. For the current subgraph $G_{i}^{\prime}\left(V_{i}, E_{i}\right)$, we define $\Delta_{\text {maxi }}^{\mid}$on the basis of procedure B and then define $\Delta_{\text {maxi }}=\Delta_{\text {maxi }}^{\mid}+\left|U_{i}\right|+$ $|U|$ and place $\Delta_{\text {maxi }}$ in the set $\mathrm{H}$, and the vertices $\mathrm{j}$ included in $\mathrm{U}$ if they exist in this set, we assign estimates equal to the maximal estimate in the set $\mathrm{H}$, and assign them to $\mathrm{H}$.

Step 5. Check the list of S for the presence of elements. If there are no items, go to step 3 , otherwise, we perform the next step.

Step 6. Select in $H=\left\{\Delta_{\max i}\right\}$ a subset of $h_{\max }=\left\{\Delta_{\max }^{*}\right\}$ upper bounds for vertices having maximal same values. Check if equality holds $\left|h_{\text {max }}\right|=\left\{\Delta_{\text {maxi }}^{*}\right\}$. If this is true, then the set of vertices for which the $\left\{\Delta_{\text {maxi }}^{*}\right\}$ bounds are obtained form the maximum size clique and the procedure finishes, otherwise we perform the next step.

Step 7. We sort out the vertices of this graph in decreasing order of the estimations $\Delta_{\text {maxi }}$, and we combine vertices with the same estimates $\Delta_{\text {maxr }}$ into subsets $\left\{\lambda^{r}\right\}$ with cardinality $\left|\lambda^{r}\right|=p_{r}$, the total number of such subsets will be denoted by $\beta$. As a result of sorting, we obtain a sequence of vertices satisfying the inequality:

$\left\{\Delta_{\max =1}\right\} \in \lambda^{r=1}>\left\{\Delta_{\operatorname{maxr}=2}\right\} \in \lambda^{r=2}>\cdots>\left\{\Delta_{r=\beta}\right\} \in \lambda^{r=\beta}$,

store it as a list $S_{r}$ and proceed to the next step.

Step 8. Using procedure B, we define a subset of vertices $r^{*}$, starting with which inequality to (7).

In this case, all the vertices in the list $S_{r}$ after $r^{*}$ are deleted from the graph $\mathrm{G}$ with edges incident to them and delete these vertices from the list $S_{r}$. Next, $S:=S_{r}$ and proceed to step 1.

\section{Example of the Problem Solution}

As an example, we find the maximum cliques in the graph $G$ shown in Figure 1. We apply the procedure A to the list of vertices of the graph $S=\{1,2,3,4,5,6,7,8\}$. None of the vertices of the graph is connected with all other vertices, therefore the set $U=\emptyset$.

Now we proceed to the formation of subgraphs $G_{i}$ corresponding vertices $\{\mathrm{i}\}$ for $\mathrm{i}=1, \ldots, 8$ of the original graph $G$. For each $G_{i}$, calculate $\Delta_{\text {maxi }}$, using $B$. We start from the vertex $\mathrm{i}=1$, which is connected with the vertices $2,3,4,5,6$. Based on these vertices, we construct the subgraph G1 in Fig. 2.

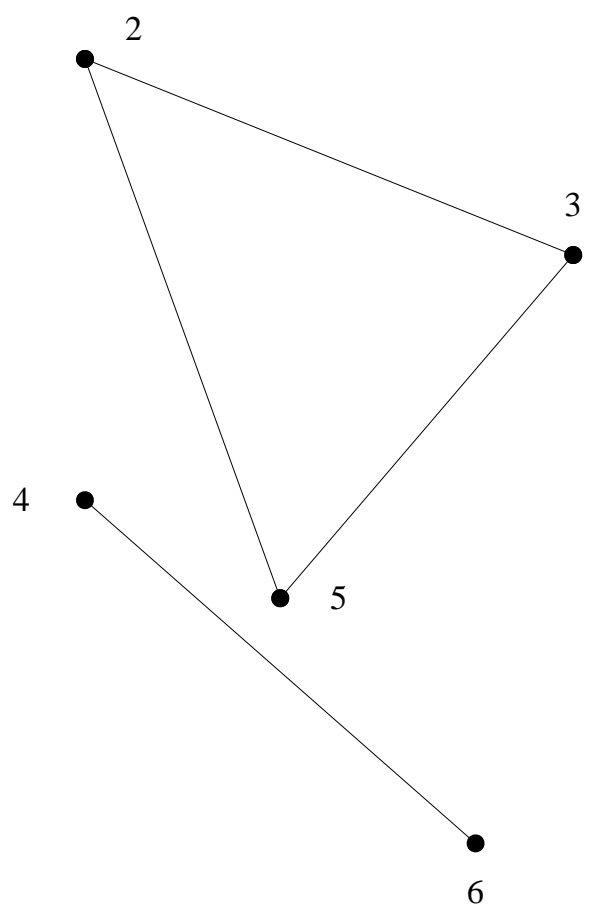

Fig. 2: Subgraph $G_{1}$.

We note that in the subgraph $G_{1}$ there are no vertices associated with all vertices of $G_{1}$. Let us specify that the subgraph $G_{1}$ is doubly-connected. Therefore, the set $U 1=\varnothing$. We sort the vertices of the graph $G_{1}$ according to formula (1). The result obtained will be placed in Table 2 .

Assign $r:=1$, investigate the subset $\alpha^{r=1}$ and check the inequality (3). In this case it has the form $0+3 \geq 2+1$. The inequality is satisfied and, consequently, according to formula $\Delta_{\max }:=d_{r}+1$ for a subgraph $G_{1} \Delta_{\max 1}:=3$.

Obviously, the calculation for the subgraphs $G_{2}, \ldots, G_{8}$ is carried out in a similar manner. We obtain the set $H=\left\{\Delta_{\max i}\right\}: \Delta_{\max 2}:=$ $3, \Delta_{\max 3}:=3, \Delta_{\max 4}:=2, \Delta_{\max 5}:=3, \Delta_{\max 6}:=2, \Delta_{\max 7}:=2$, $\Delta_{\max 8}:=8$. In this set we select the subset $h_{\text {max }}=\left\{\Delta_{\text {maxi }}^{*}\right\}$ upper bounds of vertices having maximal same values, that is $\Delta_{\max 1}:=3$, $\Delta_{\max 2}:=3, \Delta_{\max 3}:=3$, and $\Delta_{\max 5}:=3$. 
Table 2: Results of sorting the vertices of a graph $G_{1}$.

\begin{tabular}{|c|c|c|c|}
\hline \multirow{2}{*}{ Sets $\left\{\alpha^{r}\right\}$} & Vertices $i$ & $\begin{array}{c}\text { Number of } \\
\text { edges } d_{r}\end{array}$ & $\begin{array}{c}\text { Cardinality } P_{r} \text { of a } \\
\text { set }\left\{\alpha^{r}\right\}\end{array}$ \\
\hline \multirow{3}{*}{ Set $\alpha^{r=1}$} & 2 & 2 & \multirow{2}{*}{$P_{r=1}=3$} \\
\cline { 2 - 3 } & 3 & 2 & \\
\hline \multirow{2}{*}{$\operatorname{Set} \alpha^{r=2}$} & 5 & 2 & \multirow{2}{*}{$P_{r=2}=2$} \\
\cline { 2 - 3 } & 6 & 1 & \multicolumn{2}{c}{} \\
\hline
\end{tabular}

Based on this, we construct the sequence $S_{\beta}$. on $\Delta_{\text {maxi }}$ and placed in Table 3.

Table 3: Dependence of the sequence $S_{\beta}$ on $\Delta_{\operatorname{maxi}}$

\begin{tabular}{|c|c|c|c|}
\hline $\operatorname{Sets}\left\{\beta^{\gamma}\right\}$ & Vertices $i$ & $\begin{array}{l}\text { The upper bound on } \\
\text { the size of the maxi- } \\
\text { mal clique } \Delta_{\text {maxi }} \text { in } \\
\text { the graph } G_{i} \text {. }\end{array}$ & $\begin{array}{c}\text { Cardinality } \\
P_{r} \text { of a set } \\
\left\{\beta^{\gamma}\right\}\end{array}$ \\
\hline \multirow{4}{*}{ Set $\beta^{\gamma=1}$} & 1 & 3 & \multirow{4}{*}{$P_{r=1}=4$} \\
\hline & 2 & 3 & \\
\hline & 3 & 3 & \\
\hline & 5 & 3 & \\
\hline \multirow{4}{*}{ Set $\beta^{\gamma=2}$} & 4 & 2 & \multirow{4}{*}{$P_{r=2}=4$} \\
\hline & 6 & 2 & \\
\hline & 7 & 2 & \\
\hline & 8 & 2 & \\
\hline
\end{tabular}

It can be seen from the table that the inequality (7) starts to be fulfilled for $r^{*}=1$ and, therefore, the vertices $\{4,6,7,8\}$, corresponding to the values $r>1$, are removed from the initial graph $G$ and from the list. We obtain a new list $S_{\beta}=\{1,2,3,5\}$, and the graph $G$ is transformed into the graph $G^{\prime}$ (Figure 3) with a list of vertices $S=S_{\beta}$.

We apply the procedure A to the obtained graph. In Step 1 we find out that all the vertices of the graph $G^{\prime}$ are connected with all vertices of this graph $G^{\prime}$ and go to the set $U$ from the list $S=S_{\beta}$. Thus, they form a clique of the maximum size, both in the graph $G^{\prime}$, and in the graph $G$. So, the problem is solved: the maximal clique from the vertices $\{1,2,3,5\}$ in the graph $G$. Other examples of the solution of Maximum Clique Problem are considered in [8]. Application of the described method to optimize the computational components of information and control systems of railway transport will allow to optimize the computing and telecommunication resources and to refrain from premature investments in the hardware infrastructure. Reducing the number of control components of technical systems increases operational safety and extends the additional, service indicators and characteristics of railway infrastructure management systems. Methodological aspect of the logistics technologies formation in reforming processes on the railways is described in paper [9].

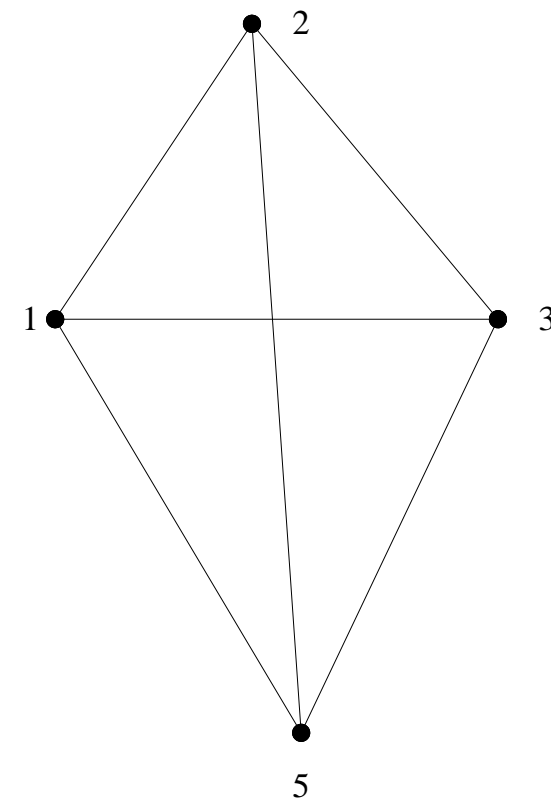

Fig. 3: Graph $G^{\prime}$ with list of vertices $S=\mathrm{S}_{\beta}$.

The problem of finding the maximum clique in an undirected graph is the key method of optimization of computing resources for distributed, parallel computations in railway transport information systems. The proposed method is advisable to use to improve a qualimetric criterion for the generalized level of vehicle [10]. In the future, it is possible to combine the examples presented in paper with the use of the method with fuzzy support system for decision-making on safety of rolling stock [11].

A model of optimization and improvement of algorithms for air fleet planning was proposed in [12], and an application of an intelligent algorithm of fuzzy regression in the modeling of freight traffic was proposed in [13]. Improvement of the technology of rolling stock management in railway transport, in work [14] is realized on the basis of an artificial neural network. The proposed mathematical model requires substantial computing resources for its implementation. These computing resources can be redistributed by optimizing the already available computer equipment in the method proposed in this article.

An interesting aspect of the application of the method can be the transport infrastructure. The influence of structural characteristics on the electrical and physical properties of electrically conductive compositions based on mineral binders is proved in [15]. At the same time, continuous improvement, modernization and cheapening of sensors for monitoring soil moisture, especially capillary type, allows continuous monitoring of microprocessor and microcontroller systems by many parameters of building objects of critical transport infrastructure [16]. In the mass application of such monitoring, it will be advisable to use network technologies to consolidate data and distribute computing resources in the network The quantitative proportions of the technical and economic indicators of the functioning of railway management systems, in particular and the safety of train traffic control, will be reported in subsequent publications. The expected effect of the innovations presented is several percent.

\section{Conclusion}

The procedure A, developed and presented, has substantive differences from the existing approaches, which use the solution of the problems of graph coloring and have exponential complexity. The proposed procedure has a small temporal complexity $O\left(2 n^{3} \log _{2} n\right)$, which allows one to solve such problems as determining the maximum clique in non-oriented graphs, determining the maximum independent sets and the minimum vertex coatings in the graphs, determining the graph isomorphism and isomorphic 
embedding. This procedure can be effectively parallelized, which will reduce the temporal complexity of its work to $O\left(2 n^{3} \log _{2} n\right)$, and as a result it will solve the complex set of problems in real time.

The introduction of procedure A for making decisions on the safety of rolling stock will reduce losses by several percent.

\section{References}

[1] Gevorkyan E., Lavrynenko S., Ruckic M., Siemiatkowski Z., Kislitsa M, "Ceramic cutting tools out of nanostructured refractory compounds", International Journal of Refractory Metals and Hard Materials, $\quad$ Vol.68, (2017), $\quad$ pp:142-144, http://dx.doi.org/10.1016/j.ijrmhm.2017.07.006

[2] Moiseenko V., Kameniev O., Gaievskyi V., "Predicting a technical condition of railway automation hardware under conditions of limited statistical data", Eastern-European Journal of Enterprise Technologies, Vol.3, No.9 (88), (2017), pp.26-35, http://dx.doi.org/10.15587/1729-4061.2017.102005

[3] IEC 61508-1:2010. Functional safety of electrical/electronic/programmable electronic safety-related systems. Part 1: General requirements, (2010)

[4] BS EN 50126-1:2017: Railway Applications. The Specification and Demonstration of Reliability, Availability, Maintainability and Safety (RAMS), Generic RAMS Process, (2017).

[5] BS EN 50128:2011: Railway Applications -Communications, signaling and processing systems. Software for Railway Control and Protection Systems, (2011).

[6] Panchenko S., Siroklyn I., Lapko A., Kameniev A., Zmii S., "Improvement of the accuracy of determining movement parameters of cuts on classification humps by methods of video analysis", Eastern-European Journal of Enterprise Technologies, Vol.4, Issue 3(82), (2016), pp.25-30, http://dx.doi.org/10.15587/17294061.2016 .76103

[7] Listrovaya E.S., Bryksin V.A., Kurtsev M.S., "Modeling Loca Scheduler Operation Based on Solution of Nonlinear Boolean Programming Problems", Cybernetics and Systems Analysis, Vol.53, No.5, (2017), pp.766-775, http://dx.doi.org/10.1007/s10559-017 9979-6

[8] Listrovoy S.V., Butenko V.M., Bryksin V.O., Golovko O.V., "Development of method of definition maximum clique in a nonoriented graph", Eastern-European Journal of Enterprise Technol $\begin{array}{llll}\text { ogies, } & \text { Vol.5, } & \text { No.4 (89), (2017), } & \text { pp.12-17, }\end{array}$ http://dx.doi.org/10.15587/1729-4061.2017.111056

[9] Lomotko D.V., Alyoshinsky E.S., Zambrybor G.G., "Methodological Aspect of the Logistics Technologies Formation in Reforming Processes on the Railways", Transportation Research Procedia, Vol.14, (2016), pp.2762-2766 http://dx.doi.org/10.1016/j.trpro.2016.05.482

[10] Panchenko S., Lavrukhin O., Shapatina O., "Creating a qualimetric criterion for the generalized level of vehicle", Eastern-European Journal of Enterprise Technologies, Vol.1, No.3 (85), (2017), pp.39-45. http://dx.doi.org/10.15587/1729-4061.2017.92203

[11] Lomotko D.V., Kovalov A., Kovalova O., "Formation of fuzzy support system for decision-making on merchantability of rolling stock in its allocation", Eastern-European Journal of Enterprise Technologies, Vol.6, No.3 (78), (2015), pp.11-17, http://dx.doi.org/10.15587/1729-4061.2015.54496

[12] Wang Y., Sun H., Zhu J., Zhu B., "Optimization Model and Algorithm Design for Airline Fleet Planning in a Multiairline Competitive Environment", Mathematical Problems in Engineering, Vol.2015, (2015), pp.1-13, http://dx.doi.org/10.1155/2015/783917

[13] Najaf P., Famili S., "Application of an Intelligent Fuzzy Regression Algorithm in Road Freight Transportation Modelling", Promet Traffic \&Transportation, Vol.25, No.4, (2013), Issue 4, pp.311-322. http://dx.doi.org/10.7307/ptt.v25i4.337

[14] Butko T.V., Prodaschuk S., Bogomazova G., Shelekhan G. Prodaschuk M., Purii R., "Improvement of technology for management of freight rolling stock on railway transport", EasternEuropean Journal Of Enterprise Technologies, No3(3), (2017), pp.4-11, http://dx.doi.org/10.15587/1729-4061.2017.103220

[15] Pluhin O., Plugin A., Plugin D., Borziak O., Dudin O., "The effect of structural characteristics on electrical and physical properties of electrically conductive compositions based on mineral binders", MATEC Web Conf., $6^{\text {th }}$ International Scientific Conference "Reliability and Durability of Railway Transport Engineering Structures and Buildings" (Transbud-2017), Vol.116, (2017), https://doi.org/10.1051/matecconf/201711601013
[16] Plugin A., Trykoz L., Herasymenko O., Pluhin A., Konev V., "Independent diagnostic computer systems with the ability to restore operational characteristics of construction facilities", Diagnostyka, Vol.19, No.2, (2018), pp.11-21, http://dx.doi.org/10.29354/diag/83009 